\title{
Risk attribution of Campylobacter infection by age group using exposure modelling
}

\author{
S. BUETTNER ${ }^{1 *}$, B. WIELAND ${ }^{2}$, K. D. C. STAERK ${ }^{2}$ AND G. REGULA ${ }^{3}$ \\ ${ }^{1}$ Federal Veterinary Office, Berne, Switzerland \\ ${ }^{2}$ Veterinary Epidemiology and Public Health, Royal Veterinary College, University of London, UK \\ ${ }^{3}$ Institute of Veterinary Public Health, Faculty of Veterinary Medicine, University of Berne, Switzerland
}

(Accepted 26 May 2010; first published online 2 July 2010)

\section{SUMMARY}

Knowledge on the relative importance of alternative sources of human campylobacteriosis is important in order to implement effective disease prevention measures. The objective of this study was to assess the relative importance of three key exposure pathways (travelling abroad, poultry meat, pet contact) for different patient age groups in Switzerland. With a stochastic exposure model data on Campylobacter incidence for the years 2002-2007 were linked with data for the three exposure pathways and the results of a case-control study. Mean values for the population attributable fractions (PAF) over all age groups and years were $27 \%$ (95\% CI 17-39) for poultry consumption, $27 \%$ (95\% CI 22-32) for travelling abroad, $8 \%$ (95\% CI 6-9) for pet contact and $39 \%$ (95\% CI 25-50) for other risk factors. This model provided robust results when using data available for Switzerland, but the uncertainties remained high. The output of the model could be improved if more accurate input data are available to estimate the infection rate per exposure. In particular, the relatively high proportion of cases attributed to 'other risk factors' requires further attention.

Key words: Campylobacter, modelling, public health, risk assessment, zoonoses.

\section{INTRODUCTION}

Human campylobacteriosis is the most commonly reported foodborne disease in Switzerland [1,2] and the European Union [3] and has become a leading cause of enteric zoonotic gastrointestinal infections in most developed and many developing countries [4]. In 2007, 6056 cases of campylobacteriosis were reported in Switzerland (83.4/100000 inhabitants) [5]. Over $90 \%$ of these infections are caused by Campylobacter jejuni, about $5 \%$ by $C$. coli and only few by other

\footnotetext{
* Author for correspondence: Dr S. Buettner, Federal Veterinary Office, Schwarzenburgstrasse 155, 3003 Bern, Switzerland. (Email: sabina.buettner@bvet.admin.ch)
}

Campylobacter spp. like C. lari or C. upsaliensis [2]. The most common symptoms are diarrhoea, abdominal pain, fever, headache, nausea, and vomitus. In most cases, no antibiotic therapy is needed because symptoms are typically self-limiting and last for 3-6 days. Post-infectious complications like reactive arthritis or Guillain-Barré syndrome are rare [6].

In Switzerland laboratories are obligated to report human cases of campylobacteriosis to the Federal Office of Public Health (FOPH). Previous studies have suggested that underreporting occurs because notification takes place if a person with campylobacteriosis visits a physician, if the physician takes a stool sample, if the pathogen is detected by a laboratory test and if the positive result is reported [7]. 
Therefore the true incidence is higher than that reported and estimates of the number of cases that occur in the community per reported case are important to estimate the total burden and cost of a disease. Estimates exist for certain countries [8-11] but are not available for Switzerland.

The most important risk factors for Campylobacter infection are: handling and consumption of raw or undercooked meat, especially poultry meat; consumption of cross-contaminated ready-to-eat food; travelling abroad; contaminated drinking water; direct contact with infected animals; and consumption of unpasteurized milk [12-20]. A case-control study in Switzerland [21] identified travelling abroad and consumption of chicken liver as the most important risk factors in adults.

To date, most efforts to reduce the risk of campylobacteriosis for humans have focused on poultry and poultry meat $[22,23]$. In some countries these measures resulted in a considerable reduction in human cases [23, 24] whereas in other countries these efforts only had a limited effect in reducing the incidence of campylobacteriosis [22]. One explanation for this may be that other sources of infection such as travel-related infection and imported poultry meat also play an important role. Knowledge on the relative importance of the different sources of human campylobacteriosis is thus important for effective prevention and control measures. Different methods can be used to assess the relative importance of different risk factors of foodborne infections, for example microbiological subtyping, case-control studies, outbreak investigations or exposure assessments [25]. The current study aimed to use existing data on exposure for different potential risk pathways and combine these data in a model that allows integration of information on each pathway. The approach does not require gathering new data and is therefore considered to be cost-effective in estimating the relative importance of different sources.

Data for Switzerland were available for the risk factors poultry meat, travel abroad and pet contact. These risk factors are among the most important factors for sporadic human campylobacteriosis [12-20, 26]. Other known risk factors were not included either because according to expert opinion these sources are not of major importance in Switzerland (raw milk, contaminated drinking or surface water) or there were not enough data available to estimate the exposure (direct contact with cattle or other animals).
Previous studies suggested that the incidence of campylobacteriosis varies according to age [1, 16, 27]. At the same time, exposure to infection sources such as travel and close contact with pets varies between age groups. This information would be useful for assessing the risk of infection through different sources. A few case-control studies estimated the importance of risk factors for younger children [12, 18], but exposure to infection sources has never been assessed separately for the different age groups.

The objective of this study was to assess the relative importance of three key sources of campylobacteriosis (travelling abroad, poultry meat, pet contact) for different age groups in Switzerland. For this, a stochastic exposure model was used which combined data on Campylobacter incidence for the years 2002-2007 with data on the three exposure pathways, the results of the Swiss case-control study and other studies describing risk factors for campylobacteriosis.

\section{MATERIALS AND METHODS}

\section{Outline of the model}

The model estimated the incidence of campylobacteriosis in five different age groups over 6 years from available data on exposure to three possible infection sources. A simple infection pathway was modelled for the exposures 'consumption of poultry meat', 'travelling abroad' and 'contact with pets' (Figs 1 and 2).

The inputs to the model were data on exposure to each source, the Campylobacter prevalence in the different sources and the estimated infection rate (IR) per exposure, which described the proportion of persons that become ill after a single exposure to a source. This IR was derived from the population attributable fraction (PAF) that was calculated from an odds ratio (OR) for the exposure that had been determined in case-control studies within a specific age group. Based on published ORs and prevalence data and assuming, that for diseases with low incidence the OR is a valid approximation of the relative risk (RR), PAF and IR were calculated as follows [28]:

$\mathrm{PAF}=[\mathrm{pE}+(\mathrm{OR}-1)] /[1+\mathrm{pE}(\mathrm{OR}-1)]$,

where $\mathrm{pE}+$ is the prevalence of Campylobacterpositive exposures within the total number of exposures to the source in the specific age group

$\mathrm{IR}=\mathrm{PAF} * n / \mathrm{E}+$, 


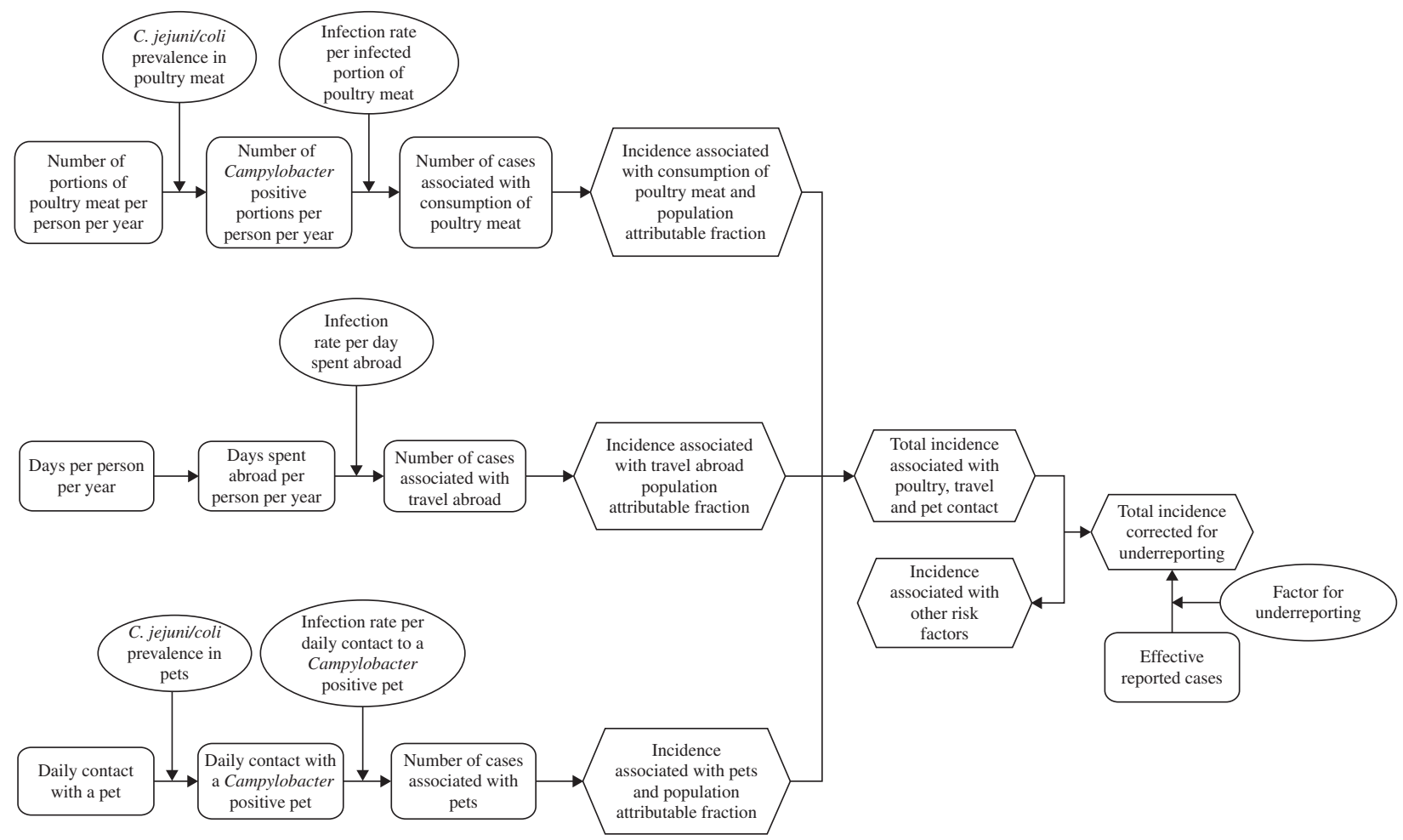

Fig. 1. Outline of a model designed to attribute Campylobacter cases to infection sources in Switzerland.

where $n$ is the total number of reported cases corrected for underreporting and $\mathrm{E}+$ is the number of exposures to a Campylobacter-positive source within the population of the specific age group.

Because it can be assumed that due to different age-dependent risk-mitigating behaviours the IR is not constant across age groups, a correction factor was introduced for the exposure pathways 'poultry consumption' (food-safety factor) and 'travelling abroad' (travel-safety factor) to adapt the IR to the different age groups.

The output of the simulation model was the incidence of Campylobacter cases/100 000 inhabitants, by year and age group associated with an exposure and the respective PAF.

Incidence $=(\mathrm{E}+* \mathrm{IR} / N) * 100000$,

where $\mathrm{E}+$ is the number of exposures to a Campylobacter-positive source within the population of the specific age group and $N$ the number of persons in this age group.

The incidence of cases attributable to other infection sources which were not included in the model was calculated as the difference between the effective incidence corrected for underreporting and the incidence associated with the three risk factors estimated by the model. If the estimated incidence was greater than

$$
\begin{aligned}
& \mathrm{I}_{\text {poultry }}=\left[\left(\mathrm{NppC} * \mathrm{IR}_{\text {poultry }}\right) / \mathrm{N}\right] * 100000 \\
& \mathrm{I}_{\text {travel }}=\left[\left(\mathrm{D} * \mathrm{IR}_{\text {travel }}\right) / \mathrm{N}\right] * 100000 \\
& \mathrm{I}_{\text {pets }}=\left\{\left[\left(\mathrm{P}_{\mathrm{c}} * \mathrm{IR}_{\mathrm{cat}}\right)+\left(\mathrm{P}_{\mathrm{d}} * \mathrm{IR}_{\mathrm{dog}}\right)\right] / \mathrm{N}\right\} * 100000 \\
& \mathrm{I}_{\text {other factors }}=\left[\left(\mathrm{I}_{\mathrm{eff}} * 1 / \mathrm{k}\right)-\left(\mathrm{I}_{\text {poultry }}+\mathrm{I}_{\text {travel }}+\mathrm{I}_{\text {pets }}\right)\right]
\end{aligned}
$$

Fig. 2. Mathematical representation of the model where $\mathrm{I}_{\text {poultry }}$ is the Campylobacter incidence due to poultry meat consumption; NppC is the number of Campylobacterpositive poultry meat portions per person per year; IR poultry is the infection rate for the consumption of a Campylobacter-positive poultry meat portion; $\mathrm{I}_{\text {travel }}$ is the incidence due to travelling abroad; $\mathrm{D}$ is the number of days spent abroad per person per year; $I_{\text {travel }}$ is the infection rate for a day spent abroad; $P_{c}$ is the prevalence of people having daily cat contact; $\mathrm{P}_{\mathrm{d}}$ is the prevalence of people having daily dog contact; $I_{R_{\mathrm{dog}}}$ is the infection rate for having daily dog contact; $\mathrm{IR}_{\mathrm{cat}}$ the infection rate for having daily cat contact; $\mathrm{I}_{\mathrm{eff}}$ is the incidence of effective reported Campylobacter cases, $\mathrm{k}$ is the factor for underreporting and $\mathrm{N}$ is the number of people at risk (Swiss population in each age group).

the effective incidence corrected for underreporting, the number of cases attributable to other sources was set to zero. A stochastic simulation model was developed using @Risk (Palisade Corp., USA)with 5000 iterations per simulation. (Supplementary material with detailed information on the model is available online.) 


\section{Input data}

\section{Population and incidence}

The population of Switzerland was stratified into five age groups based on demographic data and using reported campylobacteriosis rates from 2002 to 2007 (Table 1). Small children ( $0-4$ years), older children (5-19 years), young adults (20-34 years), adults ( $35-59$ years) and older people ( $\geqslant 60$ years) were grouped together, because it was assumed that behaviour leading to exposure in people within these groups would be similar. Another consideration that was taken into account in defining the age group was the differences in cases reported in the past and published differences in underreporting for these age groups.

Based on existing estimations of underreporting in other countries $[10,11,35]$, it was assumed that on average $15 \%$ of all cases are detected with the current reporting scheme in Switzerland. Based on expert opinion the factor for underreporting was adapted for each age group. In the young children group (0-4 years) $30 \%$ of all cases were assumed to be reported, because disease symptoms in this age group are more severe and worried parents seek medical assistance. The lowest reporting rates were assumed for the 20-34 and 35-59 years age groups. To calculate the total effective cases corrected for underreporting the under-reporting factor for 'all cases' was used (Table 2). It was also assumed that reporting of domestically acquired infections is half as likely as reporting of infections that are acquired abroad, because people who become ill after travelling abroad were assumed to be more likely to seek medical advice, and stool samples for bacteriological examination would be more likely to be taken. Therefore different factors were used for the calculation of the effective cases associated with domestic exposures and with travelling abroad. Uncertainty on the factor of underreporting was modelled using a Pert distribution (Table 2).

\section{Poultry meat consumption}

The exposure was estimated by calculating the number of Campylobacter-positive poultry meat portions consumed per person per year (Fig. 1). The Campylobacter prevalence in turkey meat is different from that in broiler meat and it is also different in meat of domestic production than in imported meat [30]. Moreover, freezing significantly lowers Campylobacter prevalence. Therefore the consumption per person per year for eight different product categories were estimated (broiler or turkey meat/fresh or frozen/import or domestic production).

The quantity of poultry meat consumed per person per year is not the same in different age groups. It was assumed that this difference was mostly due to different portion sizes and not to a different number of portions. Therefore it was concluded that the average number of portions in each age group is more or less the same and could be estimated by taking an average portion size of $150 \mathrm{~g}$ across all age groups.

The prevalence of $C$. jejuni and C. coli on poultry meat was estimated from data on the Campylobacter prevalence on broilers in the slaughterhouse (years 2002-2006) and on broiler meat at retail (2007). The prevalence of Campylobacter on turkey meat was estimated from a prevalence study at retail in 2005 [30]. The apparent prevalence (AP) was corrected with the test specificity (Sp) and test sensitivity (Se) to estimate the true prevalence (TP) using the following formula [28]:

$\mathrm{TP}=(\mathrm{AP}+\mathrm{Sp}-1) /(\mathrm{Se}+\mathrm{Sp}-1)$.

The IR was estimated based on the OR for poultry meat consumption from a Swiss case-control study [21]. With the OR the PAF for the 20-34 and 35-59 years age groups was calculated and based on this the IR per portion of consumed Campylobacterpositive poultry meat for these age groups was derived. The minimum, mean, and maximum values of IR for poultry consumption calculated for the years 2002-2007 were used to define the Pert distribution reflecting the uncertainty of a general IR for poultry consumption.

Various studies reported that especially young adults (19-34 years) are at higher risk of Campylobacter infection probably because of inappropriate kitchen hygiene [31, 36], which should result in different IR for poultry meat consumption in different age groups. In our model, the estimation of the IR was based on data of the young and middle-aged adults (19-34 and 35-59 years, respectively). Therefore this rate had to be adapted with a food-safety factor for the different age groups which was based on expert opinion on estimated kitchen hygiene in each group. The $0-4$ years age group was considered to have the lowest risk (i.e. high kitchen hygiene) with the 20-34 years age group having the highest risk which was reflected in the applied food-safety factors (Table 3). Uncertainty of the values of the food-safety factor was modelled using a Pert distribution. 
Table 1. Data sources used for modelling the exposure

Data $\quad$ Source

Remarks

Population

Number of persons per age group

Swiss Federal Statistical Office (SFSO), yearly reports on the permanent resident population in Switzerland (2002-2007)

Reported cases

Poultry meat consumption

Total poultry meat production

Poultry sales at retail

Poultry sales at whole sale

Total poultry meat production

Total imported poultry

Portion size

Campylobacter prevalence on broiler meat

Campylobacter prevalence in broiler herds

Campylobacter prevalence on turkey meat

Test sensitivity and specificity

OR poultry meat consumption

Travelling abroad

Proportion of population with at least one trip

per year with at least one overnight stay

(net travel propensity) per age group

Mean number of trips per travelling person

(travel frequency)

Duration of trips

Proportion of trips in Switzerland and trips abroad for short trips

Proportion of trips in Switzerland and trips

abroad for long trips

OR travelling abroad

\section{Pet contact}

Percent households with cats and dogs

Federal Office of Public Health (FOPH), Bulletin

(2002-2007)

Swiss Farmers' Union, Statistik Viehwirtschaft (2002-2007) IHA-GFK, Hergiswil (scanning data of all relevant

Divided into broiler and turkey meat

supermarkets in Switzerland)

Data was available for the years 2004 to 2007. In the years 2002/2003 the

values of 2004 were used

Prodega, personal communication

Proviande, yearly statistics of meat market

Swiss Federal Customs Administration, Swiss Impex,

statistics on foreign trade

[29]

FVO, unpublished monitoring data

A. G. Bell, personal communication

[30]

$[31]$

[21]

$[32,33]$

SFSO (2005/2007) Reiseverhalten der Schweizer Wohnbevölkerung 2003/2005

SFSO (2005/2007) Reiseverhalten der Schweizer Wohnbevölkerung 2003/2005

SFSO (2005/2007) Reiseverhalten der Schweizer

Wohnbevölkerung 2003/2005

Divided into fresh and frozen

Data was available only for 2007, same data was used for the other years

Divided into imports and domestic production

Divided into fresh and frozen and broiler and turkey meat

Data estimated from prevalence at slaughterhouse in 2006 and at retail in 2007

Data estimated from prevalence at the slaughterhouse 2002-2005

Data only for 2005

Age 15-64 yr

For 2002/2003 same percentages were assumed as for 2004, because net travel propensity in 2001 was the same as in 2004; for 2005 and 2006 a linear increase in net travel propensity was assumed

Data was available for the years 2001, 2004 and 2007. In the years 2002/2003 and 2005/2006 a linear decrease or increase in the number of trips was assumed

Duration of trips has remained more or less stable over the last 10 years

(Travel Market Switzerland, 2007)

Data for 2003 was used for the years 2002 and 2004; data of 2005 was used for 2006 and 2007

Data for 2003 was used for the years 2002 and 2004; data of 2005 was used for 2006 and 2007

Age group 15-64 yr

S. A. Mars, personal communication

Data were available for 2004 and 2007, the percentages stayed the same over the years (S. A. Mars, personal communication)

Data only for $2001 / 2002$

All age group

in dogs and cats

Test sensitivity and specificity

Expert opinion

OR pet contact

[16] 
Table 2. Estimated proportion of Campylobacter cases that are reported in different age groups and with different origin of infection (domestic or travel associated) in Switzerland. These proportions were used to correct the reported Campylobacter incidence for underreporting

\begin{tabular}{llll}
\hline \hline $\begin{array}{l}\text { Age group } \\
\text { (yr) }\end{array}$ & All cases & Domestic cases & Travel associated \\
\hline $0-4$ & $\operatorname{Pert}(0 \cdot 25,0 \cdot 3,0 \cdot 35)$ & $\operatorname{Pert}(0 \cdot 15,0 \cdot 2,0 \cdot 25)$ & $\operatorname{Pert}(0 \cdot 4,0 \cdot 45,0 \cdot 5)$ \\
$5-19$ & $\operatorname{Pert}(0 \cdot 09,0 \cdot 11,0 \cdot 15)$ & $\operatorname{Pert}(0 \cdot 05,0 \cdot 075,0 \cdot 125)$ & $\operatorname{Pert}(0 \cdot 125,0 \cdot 15,0 \cdot 175)$ \\
$20-34$ & $\operatorname{Pert}(0 \cdot 05,0 \cdot 08,0 \cdot 11)$ & $\operatorname{Pert}(0 \cdot 01,0 \cdot 05,0 \cdot 09)$ & $\operatorname{Pert}(0 \cdot 08,0 \cdot 11,0 \cdot 14)$ \\
$35-59$ & $\operatorname{Pert}(0 \cdot 06,0 \cdot 09,0 \cdot 12)$ & $\operatorname{Pert}(0 \cdot 04,0 \cdot 07,0 \cdot 1)$ & $\operatorname{Pert}(0 \cdot 09,0 \cdot 12,0 \cdot 15)$ \\
$\geqslant 60$ & $\operatorname{Pert}(0 \cdot 12,0 \cdot 15,0 \cdot 18)$ & $\operatorname{Pert}(0 \cdot 09,0 \cdot 12,0 \cdot 15)$ & $\operatorname{Pert}(0 \cdot 125,0 \cdot 175,0 \cdot 2)$ \\
\hline \hline
\end{tabular}

Table 3. Factors used to correct the estimated Campylobacter infection rates for poultry consumption ('food safety') and travelling abroad ('travel safety'). Different infection rates were used in different age groups to correct for differences in risk behaviour

\begin{tabular}{lll}
\hline \hline $\begin{array}{l}\text { Age group } \\
(\mathrm{yr})\end{array}$ & Food-safety factor & Travel-safety factor \\
\hline $0-4$ & $\operatorname{Pert}(0 \cdot 2,0 \cdot 25,0 \cdot 3)$ & $\operatorname{Pert}(0 \cdot 35,0 \cdot 4,0 \cdot 45)$ \\
$5-19$ & $\operatorname{Pert}(0 \cdot 55,0 \cdot 6,0 \cdot 65)$ & $\operatorname{Pert}(0 \cdot 45,0 \cdot 5,0 \cdot 55)$ \\
$20-34$ & $\operatorname{Pert}(1 \cdot 2,1 \cdot 25,1 \cdot 30)$ & $\operatorname{Pert}(1 \cdot 1,1 \cdot 15,1 \cdot 2)$ \\
$35-59$ & $\operatorname{Pert}(0 \cdot 6,0 \cdot 65,0 \cdot 7)$ & $\operatorname{Pert}(0 \cdot 65,0 \cdot 7,0 \cdot 75)$ \\
$\geqslant 60$ & $\operatorname{Pert}(0 \cdot 45,0 \cdot 5,0 \cdot 55)$ & $\operatorname{Pert}(0 \cdot 5,0 \cdot 55,0 \cdot 6)$ \\
\hline \hline
\end{tabular}

The number of cases associated with poultry consumption was calculated by multiplying the number of consumed infected portions per age group with the IR corrected with the food-safety factor for each age group and the number of persons in this age group.

\section{Travelling abroad}

Exposure was estimated by assessing the number of days spent abroad per year for each age group (Fig. 1). Statistics on days spent abroad were obtained from data of the Swiss Federal Statistical Office (SFSO) and data published by the Institute of Public Services and Tourism of the University of St Gallen (Table 1). The trips were stratified into short $(<4$ overnight stays) and long ( $\geqslant 4$ overnight stays) trips. Based on the proportions of travel abroad for short and long trips and the mean duration of a short and a long trip in the different age groups, the total days spent abroad were calculated for each year.

The OR for travelling abroad from a Swiss casecontrol study [21] was used to derive the IR per day abroad for the 20-34 and 35-59 years age groups. The prevalence of travel exposure for these age groups was calculated by dividing the days spent abroad by the total days per year in these age groups. The minimum, mean, and maximum values of the IRs for travelling abroad calculated for the years 2002-2007 were used to define a Pert distribution as an input for the IR per day abroad used in the model.

It was assumed that IR per day abroad was not the same in different age groups, because young adults especially were assumed to follow hygienic recommendations for travellers less strictly than persons from other age groups. In our model, the estimation of the IR was based on data of the young and middle-aged adults (19-34 and 35-59 years, respectively). Therefore this rate had to be adapted with a travel-safety factor for the different age groups according to expert opinion. Uncertainty of the values of the travel-safety factor was modelled using a Pert distribution (Table 3). The number of cases associated with travelling abroad was calculated by multiplying the number of days spent abroad in this age group with the IR corrected with the travel-safety factor for this age group.

\section{Contact with pets}

Exposure was estimated by assessing the prevalence of persons in each age group with daily contact with an infected cat or dog (Fig. 1). Data on the penetration of dogs and cats in households of different categories (young families with children, young families with teenagers, adult families, young couples without children, older couples without children, young singles, older singles) were obtained from a pet-nutrition company, which conducts a yearly telephone survey on this matter. By estimating the percentage of persons in the different age groups belonging to the given household categories, the number of persons with a pet in the same household was calculated (daily dog/cat contact).

Prevalence data of $C$. jejuni and C. coli in cats and dogs, corrected for the test sensitivity and specificity, 
were used to calculate the number of persons with daily contact with an infected pet [34].

To estimate the IR for a daily contact with a pet, the OR data for cat and dog contact of a Swedish case-control study [16] were used. The IR for daily cat/dog contact for the years 2002-2007 were calculated and the minimum, mean, and maximum values of this output were used to define a Pert distribution to derive the average IR for daily cat/dog contact.

It was assumed that IR was the same in all age groups. The number of cases associated with daily cat/dog contact was calculated by multiplying the number of persons with daily cat/dog contact with the IR for daily cat/dog contact.

\section{Other risk factors}

The incidence due to risk factors not included in the model was calculated by subtracting the estimated incidences due to poultry consumption, travelling abroad and pet contact from the total incidence corrected for underreporting (Fig. 2). To estimate the total reported cases, the estimated reported cases associated with poultry consumption, travelling abroad and pet contact were subtracted from the total effective reported cases and the means of these differences over the years for every age group were calculated. For each age group these values were defined as model outputs and the minimum (truncated at 0), mean, and maximum of these outputs were used to define a Pert distribution for the mean of the reported cases associated with other risk factors.

\section{Sensitivity analysis}

The effect of uncertainty and variability of input variables on the model outputs 'incidence associated with poultry consumption', 'incidence associated with travelling abroad', 'incidence associated with pet contact' and 'total incidence' was assessed using the sensitivity analysis tool of the@Risk software. With multivariate stepwise regression the standardized $\beta$ coefficients for the associated input variables of an output were calculated. The most influential variables were determined by the magnitude of the standardized $\beta$ coefficients.

\section{RESULTS}

\section{Exposure to risk factors}

Campylobacter prevalence of broiler meat decreased from $53.8 \%(95 \%$ CI $49 \cdot 8-58 \cdot 2)$ in 2002 to $27 \cdot 5 \%$

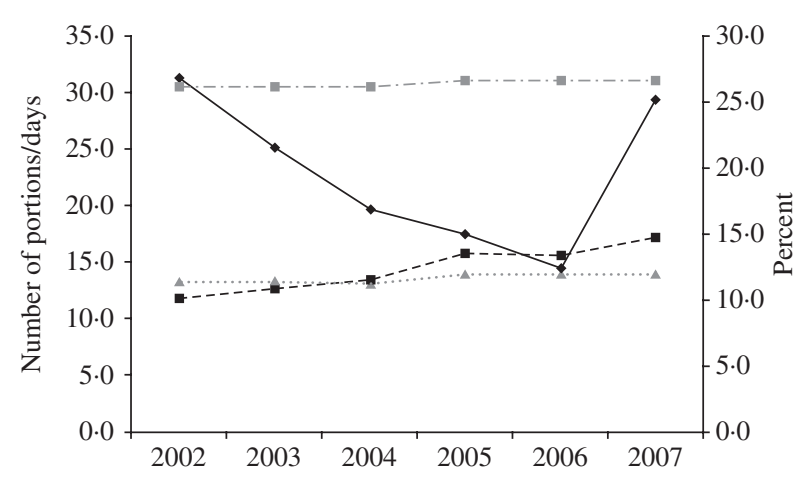

Fig. 3. Number of Campylobacter-positive poultry meat portions consumed per person per year $(--)$, days spent abroad per person per year (- - - - -) and percent of population with daily cat contact (-- ) or dog contact $(\cdots \ldots)$ for the years 2002-2007 in Switzerland.

(95\% CI 25.4-29.7) in 2006 and then increased again in 2007 to $52 \%(95 \%$ CI $41 \cdot 2-63 \cdot 7)$.

Poultry consumption also decreased from $9.7 \mathrm{~kg}$ per capita per year to $8.2 \mathrm{~kg}$ per capita per year in 2006 and increased again in 2007 to $9 \cdot 5 \mathrm{~kg}$.

Therefore the number of estimated consumed Campylobacter-positive portions per person per year halved from $31 \cdot 2(95 \%$ CI $25 \cdot 0-39 \cdot 4)$ in 2002 to $14 \cdot 2$ $(95 \%$ CI $11 \cdot 5-18 \cdot 3)$ in 2006 and then increased again to $29 \cdot 3$ (95\% CI $22 \cdot 9-37 \cdot 6$ ) in 2007 (Fig. 3).

The number of days spent abroad steadily increased from 2002 to 2007 in all age groups (Fig. 3). Small children spent the fewest and young adults (20-34 years) the most days abroad. In small children the number of days spent abroad increased from 6.5 (95\% CI 5.8-7.0) to $9 \cdot 5(95 \%$ CI $8 \cdot 7-10 \cdot 4)$ and in young adults from $13 \cdot 9(95 \% \mathrm{CI} 12 \cdot 6-15 \cdot 3)$ to $23 \cdot 1$ (95\% CI 20.8-25.4), respectively.

The prevalence of daily cat/dog contact remained constant in all age groups (Fig. 3) and was highest in children aged 5-19 years with $33 \%$ of the people in this age group having daily cat contact and $17.4 \%$ having daily dog contact. The prevalence of daily cat $/$ dog contact was least in older people, with $22.7 \%$ having daily cat contact and $14 \%$ having daily dog contact.

\section{Model outputs}

The model outputs for PAF for the different exposure pathways in the different age groups from 2002 to 2007 are shown in Table 4 . Travelling abroad and poultry consumption were both responsible for $19 \%$ (mean values cited), respectively, of the 
Table 4. Population attributable fractions for Campylobacter infection due to poultry consumption, travelling abroad, pet contact and other risk factors in five different age groups in Switzerland for the years 2002-2007

\begin{tabular}{|c|c|c|c|c|c|c|c|c|c|c|c|c|c|c|}
\hline \multirow{2}{*}{$\begin{array}{l}\text { Age } \\
\text { group } \\
\text { (yr) }\end{array}$} & \multicolumn{2}{|c|}{2002} & \multicolumn{2}{|c|}{2003} & \multicolumn{2}{|c|}{2004} & \multicolumn{2}{|c|}{2005} & \multicolumn{2}{|c|}{2006} & \multicolumn{2}{|c|}{2007} & \multicolumn{2}{|c|}{ Total } \\
\hline & M & $95 \% \mathrm{CI}$ & M & $95 \% \mathrm{CI}$ & M & $95 \% \mathrm{CI}$ & M & $95 \% \mathrm{CI}$ & M & $95 \% \mathrm{CI}$ & M & $95 \% \mathrm{CI}$ & M & $95 \% \mathrm{CI}$ \\
\hline \multicolumn{15}{|c|}{ Poultry consumption } \\
\hline $0-4$ & 21 & $13-31$ & 21 & $13-32$ & 17 & $10-25$ & 16 & $10-24$ & 13 & $8-20$ & 27 & $17-41$ & 19 & $12-28$ \\
\hline $5-19$ & 34 & $21-50$ & 34 & $21-50$ & 25 & $15-37$ & 25 & $15-37$ & 20 & $12-30$ & 37 & $22-55$ & 29 & $18-43$ \\
\hline $20-34$ & 27 & $16-42$ & 25 & $15-39$ & 22 & $13-33$ & 21 & $12-32$ & 17 & $10-26$ & 31 & $18-48$ & 24 & $14-37$ \\
\hline $35-59$ & 29 & $18-44$ & 28 & $17-43$ & 23 & $14-35$ & 21 & $13-32$ & 18 & $11-26$ & 31 & $19-47$ & 25 & $15-38$ \\
\hline$\geqslant 60$ & 49 & $31-72$ & 42 & $26-62$ & 32 & $20-47$ & 28 & $18-41$ & 24 & $15-36$ & 41 & $25-61$ & 36 & $23-53$ \\
\hline \multicolumn{15}{|c|}{ Travelling abroad } \\
\hline $0-4$ & 12 & $10-15$ & 17 & $13-20$ & 18 & $14-21$ & 22 & $18-27$ & 22 & $18-27$ & 25 & $20-31$ & 19 & $15-24$ \\
\hline $5-19$ & 15 & $12-19$ & 21 & $16-26$ & 21 & $16-26$ & 28 & $22-35$ & 26 & $20-33$ & 26 & $20-33$ & 23 & $18-29$ \\
\hline $20-34$ & 20 & $15-26$ & 25 & $18-33$ & 29 & $21-37$ & 39 & $28-50$ & 39 & $28-50$ & 40 & $29-52$ & 32 & $23-42$ \\
\hline $35-59$ & 21 & $16-27$ & 28 & $21-36$ & 31 & $23-40$ & 38 & $29-49$ & 36 & $27-47$ & 34 & $26-44$ & 32 & $24-41$ \\
\hline$\geqslant 60$ & 26 & $20-32$ & 29 & $23-37$ & 30 & $23-37$ & 31 & $24-38$ & 30 & $24-37$ & 27 & $21-33$ & 29 & $23-36$ \\
\hline \multicolumn{15}{|c|}{ Pet contact } \\
\hline 0-4 & 8 & $6-10$ & 10 & $8-12$ & 10 & $8-12$ & 12 & $9-14$ & 12 & $9-14$ & 12 & $9-14$ & 10 & $8-13$ \\
\hline $5-19$ & 8 & $6-10$ & 10 & $7-12$ & 9 & $7-12$ & 10 & $8-13$ & 10 & $8-13$ & 9 & $7-12$ & 9 & $7-12$ \\
\hline $20-34$ & 2 & $2-3$ & 2 & $2-3$ & 3 & $2-4$ & 03 & $2-4$ & 3 & $2-4$ & 3 & $2-03$ & 3 & $2-3$ \\
\hline $35-59$ & 5 & $4-7$ & 6 & $5-8$ & 6 & $5-8$ & 07 & $5-9$ & 7 & $5-9$ & 6 & $4-8$ & 6 & $5-8$ \\
\hline$\geqslant 60$ & 10 & $8-12$ & 11 & $8-13$ & 10 & $8-13$ & 11 & $8-13$ & 11 & $9-14$ & 9 & $7-11$ & 10 & $8-13$ \\
\hline \multicolumn{15}{|c|}{ Other risk factors } \\
\hline $0-4$ & 60 & $48-69$ & 52 & $40-62$ & 55 & $45-64$ & 50 & $40-59$ & 53 & $44-61$ & 36 & $20-49$ & 51 & $40-61$ \\
\hline $5-19$ & 44 & $25-58$ & 36 & $17-52$ & 45 & $30-58$ & 37 & $21-51$ & 44 & $29-56$ & 29 & $09-46$ & 39 & $21-53$ \\
\hline $20-34$ & 50 & $33-65$ & 47 & $29-62$ & 47 & $30-62$ & 38 & $19-55$ & 42 & $24-57$ & 28 & $07-48$ & 42 & $23-58$ \\
\hline $35-59$ & 44 & $26-60$ & 37 & $18-54$ & 39 & $21-55$ & 33 & $15-50$ & 39 & $23-54$ & 29 & $09-47$ & 37 & $18-53$ \\
\hline$\geqslant 60$ & 19 & $03-37$ & 20 & $04-37$ & 29 & $12-44$ & 31 & $14-45$ & 35 & $20-48$ & 25 & $06-42$ & 25 & $5-42$ \\
\hline
\end{tabular}

M, Mean; CI, confidence interval.

Campylobacter cases in small children. Ten percent of the cases could be associated with direct pet contact and $51 \%$ were due to other risk factors.

In the older children group (5-19 years) $29 \%$ of the cases were attributable to poultry consumption. The percentage of cases due to travelling abroad in this age group was $23 \%$. Nine percent of the cases were associated with pet contact and $39 \%$ with other risk factors.

In the adult age groups (20-34 and 35-59 years) PAF for poultry consumption was $24 \%$ and $25 \%$, respectively. The percentage of cases associated with travelling abroad was $32 \%$ in both age groups. Pet contact was associated with $3 \%$ of the cases in the $20-34$ years age group and $6 \%$ in the 35-59 years age group. The percentage of cases that could be attributed to other risk factors was $42 \%$ for the 20-34 years age group and $37 \%$ for the 35-59 years age group.

In elderly people PAF for poultry consumption was $36 \%$. Twenty-nine percent of the cases were associated with travelling abroad and $10 \%$ with pet contact. In this age group $25 \%$ of the cases could be attributed to other risk factors.

Mean PAF values over all age groups and years were $27 \%$ (95\% CI 17-39) for poultry consumption, $27 \%$ (95\% CI $22-32 \%$ ) for travelling abroad, $8 \%$ (95\% CI 6-9) for pet contact and 39\% (95\% CI 25-50\%) for other risk factors (Fig. 4).

From 2002 to 2007 incidence due to travel abroad increased from $807 \cdot 4$ to 1211.7 cases/100 000 inhabitants. Incidence associated with poultry consumption first decreased from $1271 \cdot 3$ to $588 \cdot 7$ cases/100 000 in 2006 and then increased again to $1994 \cdot 1$ in 2007. Incidence due to pets remained stable with 211.9 cases in 2002 and 219.9/100 000 in 2007, whereas incidence due to other factors constantly decreased from $2013 \cdot 2$ to $1093 \cdot 9 / 100000$ inhabitants.

As seen in Figure 5 the highest mean Campylobacter incidence after correcting for underreporting was found in the 20-34 years age group with a mean incidence of 1512.2 cases/100000 inhabitants per 


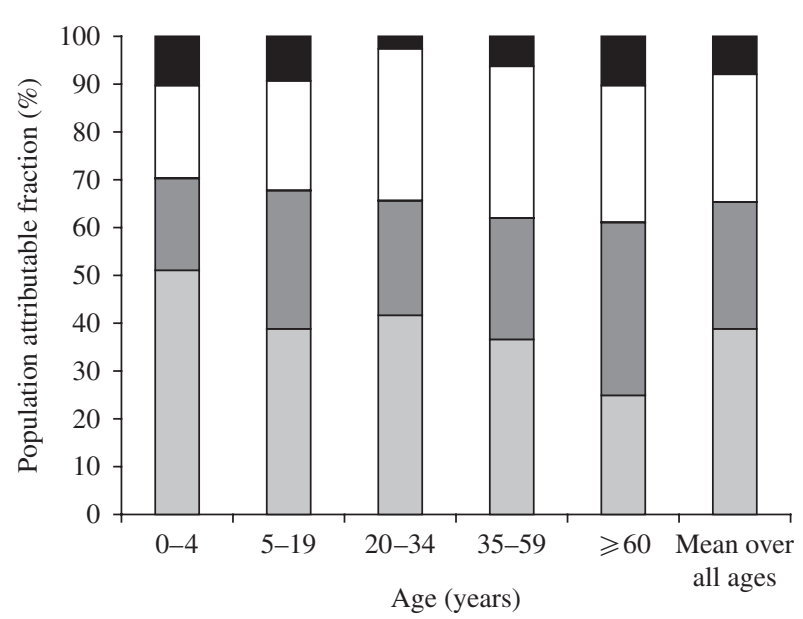

Fig. 4. Population attributable fraction (mean over the years 2002-2007) for pet contact ( $\boldsymbol{\square})$, travelling abroad ( $\square$ ), poultry consumption ( $\square$ ) and other risk factors ( $\square$ ) in five age groups in Switzerland.

year. In small children, the mean estimated incidence was 372.9 cases $/ 100000$ inhabitants per year.

\section{Sensitivity analyses}

The uncorrected IR per portion of poultry meat and the portion size had the biggest influence on the model outcome 'incidence associated with poultry consumption', with $\beta$ coefficients in the different age groups ranging from 0.61 to 0.72 and -0.53 to -0.64 , respectively. The correction factor for poultry consumption and the test sensitivity were also significantly influencing this outcome with $\beta$ coefficients ranging from $-0 \cdot 14$ to $-0 \cdot 21$ and $0 \cdot 24$ to $0 \cdot 34$, respectively. The influence of Campylobacter prevalence in broiler meat varied between meat categories with $\beta$ coefficients from $0 \cdot 17$ to 0.24 for prevalence on frozen broiler meat and 0.06 to $0 \cdot 15$ for prevalence on chilled broiler meat.

The uncorrected estimated IR per day abroad had the greatest influence on incidence of Campylobacter cases associated with travelling abroad with $\beta$ coefficients ranging from 0.88 to 0.92 , followed by the mean duration of travels with 4-7 overnight stays ( $\beta$ coefficients $0 \cdot 24-0 \cdot 4$ ) and the different correction factors for age groups ( $\beta$ coefficients $0 \cdot 1-0 \cdot 28$ ). In adult age groups (20-34 and 35-59 years) the mean duration of travels with $31-60$ overnight stays had a major influence on the incidence associated with travelling with $\beta$ coefficients of 0.23 and 0.34 , respectively.

The model outcome 'incidence associated with pet contact' was mostly influenced by the IR for daily

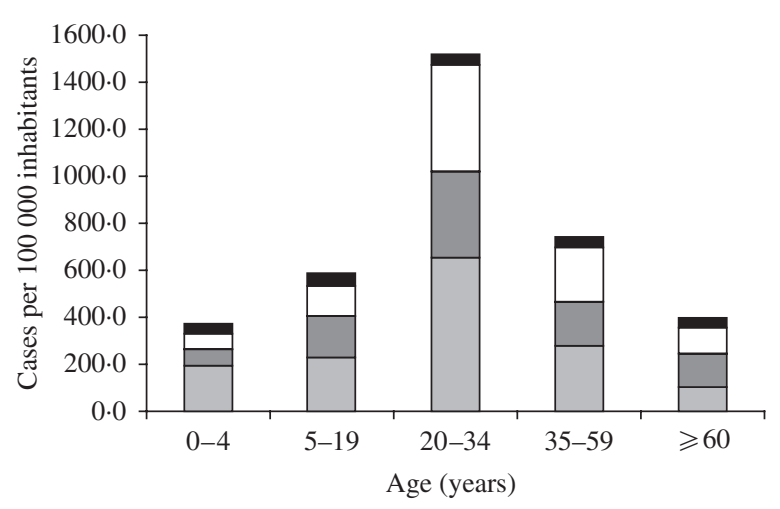

Fig. 5. Estimated incidence of Campylobacter cases corrected for underreporting (mean over the years 2002-2007) due to pet contact ( $\mathbf{\square})$, travelling abroad $(\square)$, poultry consumption $(\square)$ and other risk factors $(\square)$ in five age groups in Switzerland.

$\operatorname{dog} /$ cat contact with $\beta$ coefficients ranging from $0 \cdot 61$ to $0 \cdot 83$.

The total reported incidence was mostly influenced by the uncorrected IR per portion of poultry meat ( $\beta$ coefficient $0 \cdot 44$ ), the mean estimated reported cases associated with other risk factors for age groups 20-34 years ( $\beta$ coefficient $0 \cdot 39$ ) and $35-59$ years ( $\beta$ coefficient $0 \cdot 38$ ), the uncorrected IR per day abroad ( $\beta$ coefficient $0 \cdot 36$ ), the portion size ( $\beta$ coefficient $-0 \cdot 26)$ and the mean estimated reported cases associated with other risk factors for age groups $\geqslant 60$ years $(\beta$ coefficient $0 \cdot 23)$ and $5-19$ years $(\beta$ coefficient $0 \cdot 18$ ). Other influential factors include the factors for underreporting in age groups $20-34$ years ( $\beta$ coefficient $0 \cdot 17$ ) and $35-39$ years $(\beta$ coefficient $0 \cdot 14)$, and test sensitivity ( $\beta$ coefficient $-0 \cdot 1)$.

\section{DISCUSSION}

Based on source-attribution modelling, the relative importance of three key exposure pathways for human campylobacteriosis in different age groups was estimated over a period of 6 years.

The outputs of the model indicate that there are considerable differences between age groups. This should be taken into account in future information campaigns depending on the target audience. The age group where the fewest cases could be attributed to any of the risk factors explored were small children. There are several case-control studies that show that the risk factors for small children are different from those for adults $[12,17,18]$. Moreover, a genotyping study conducted in Scotland [37] showed that small children in rural regions had a higher risk for 
campylobacteriosis than those in urban regions and that young children in urban areas were more likely to be infected with poultry-associated Campylobacter strains, whereas children in rural regions were more likely to be infected with ruminant-associated strains. In Switzerland there are no specific studies on risk factors for small children and more research is needed to address this important knowledge gap.

Over all age groups and over all years of our analysis, a mean proportion of $27 \%$ (95\% CI 17-39) of cases were attributed to poultry consumption indicating that this source of infection is still of major importance in Switzerland. This is in accord with the results of several case-control studies from other countries that found proportions of cases associated with the consumption of chicken meat ranging from $23 \cdot 8$ to $29 \cdot 3 \%[13,15,38,39]$. Recent source attribution studies based on the multilocus sequence typing method (MLST) from England, Scotland and New Zealand estimated that $50-80 \%$ of human cases were related to the chicken reservoir [37, 40-42]. This difference can, on the one hand, be explained by the fact that part of the cases that are due to travel abroad are associated with poultry consumption in a foreign country and with genotyping methods would be associated with poultry. On the other hand some of the MLST studies exclude cases associated with travel, which results directly in a higher percentage of cases associated with poultry. Additionally, strains from the poultry reservoir may infect humans not only by the food pathway but also by the environment or by direct contact. These pathways were not considered in our model. A comparison between poultry meat source attribution based on case-control and genotyping studies suggested that differences could be due to an overestimation by genotyping because of yet incomplete data on other reservoirs than farm animals and also due to an underestimation by casecontrol studies because of misclassification due to immunity, resulting in exposed people not becoming ill [43].

The output of our model indicated that if the Campylobacter prevalence on poultry meat could be lowered to zero, $27 \%$ of the human Campylobacter cases could potentially be prevented. Campylobacter spp. is widespread in the environment and a common component of the avian gut flora. It is therefore very difficult to prevent chickens from becoming colonized and even stringent biosecurity measures cannot predictably keep campylobacters out of a poultry flock $[44,45]$. Therefore, only a reduction of the level of contamination may be achievable and this will only be possible if stringent safety measures are applied throughout the whole food-production chain.

Our finding of $27 \%$ (95\% CI 22-32) of cases attributable to travel appears to be high compared to estimates in other studies [12-14] but is in accord with results of a recent case-control study from the UK, where $24 \%$ of cases travelled abroad in the previous 14 days [46], and a meta-analysis of 37 case-control studies on risk factors for campylobacteriosis, that found international travel to be the most important risk factor for sporadic campylobacteriosis [25]. The importance of this risk factor in a population is not only dependent on travel activities and duration but also on preferences in travel destinations. The risk for Campylobacter infection is higher for travel to Asia and Africa than for travel within Europe [47] and the relative importance of this pathway is also dependent on the proportion of cases that are infected in the country of residence. Our model did not take travel destination into account because there were no data available on the association between travel destination and the risk of campylobacteriosis infection for Switzerland. The estimated importance of travel for elderly people is high, with $29 \%$ of cases associated with this source of infection. This could be due to the fact that in Switzerland elderly people travel frequently, with a proportion of $82-89 \%$ having at least one trip with at least one overnight stay [33]. Our results suggest that comprehensive information about hygienic behaviour for travellers is still of major importance for the prevention of Campylobacter cases in Switzerland.

Our model estimated that $10 \%$ of the Campylobacter cases in small children and in elderly people are due to daily pet contact, whereas in young and middle-aged adults only $3 \%$ and $6 \%$, respectively, were attributed to this infection pathway. These findings are in agreement with other studies that estimated $2 \cdot 9-7 \%$ of the cases were due to frequent contact with dogs or cats $[38,39]$. There are no known interventions that could lower the prevalence of Campylobacter in pets, therefore the only measure to lower the risk from this source is to promote hygiene in contact with animals.

Recent source attribution studies based on microbial subtyping suggested that ruminants, especially cattle and sheep, were a major source for human campylobacteriosis [37, 40-42]. This source was not included here because a genotyping study from Switzerland suggested that isolates likely to originate 


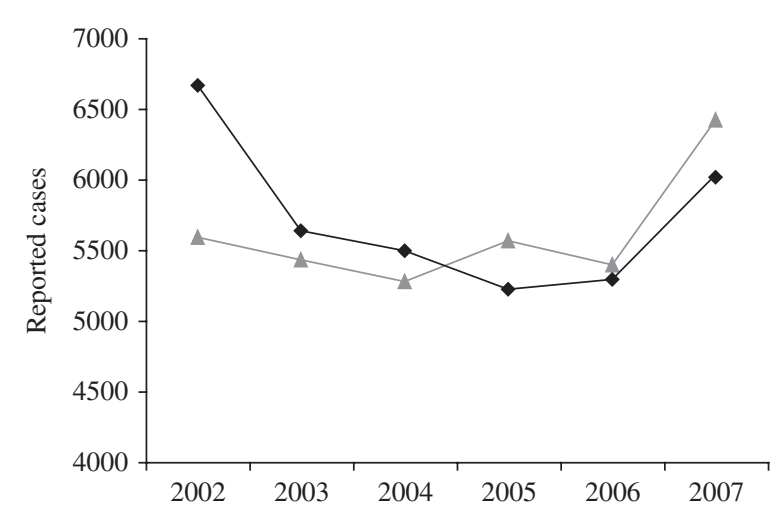

Fig. 6. Comparison of the reported Campylobacter cases in Switzerland $(--)$ ) and the estimated reported cases using the risk attribution model (__ ) for the years 2002-2007.

from cattle accounted for $<10 \%$ [48]. Meat from ruminants and raw milk cheese is generally considered to be of low risk $[49,50]$ and raw milk is only rarely consumed in Switzerland. However, occupational and environmental exposure pathways for ruminants might be relevant and should be investigated.

Figure 6 shows a comparison of the total reported cases per year estimated with the model and the effective reported cases per year in Switzerland. For 2002 the model underestimated the cases, whereas for the years 2005-2007 the cases were slightly overestimated. Regarding the relative importance of sources over time, our results suggest that the decrease in reported Campylobacter cases from 2002 to 2006 was mainly due to a decrease in exposure by poultry meat. Campylobacter prevalence on broiler meat and poultry consumption decreased in this time period and then increased again in 2007. This finding again highlights the importance of this exposure pathway.

The amount of cases due to other risk factors differs from year to year, with a decrease in four of the five age groups. This risk factor covers the residual proportion of cases that could not be attributed to other sources. It is therefore sensitive to changes in the other risk pathways. Changes between years could be due to one or several of the following reasons. The input variable 'Campylobacter prevalence in broiler meat' is based on data from different sources. Different sampling strategies or different laboratory methods could have influenced the apparent prevalence in different ways. Due to lack of information these differences could not be accounted for in the model. Another possible explanation for the different amounts of cases due to other risk factors is an effective change in the exposure to other sources. Multiple sources with changing Campylobacter prevalence over the years, for example surface water or other animal and food sources, could be responsible for these differences.

One limitation of the model is that it did not take immunity into account while epidemiological evidence suggests that infection with Campylobacter can induce protective immunity, not only in highly exposed humans in developing countries but also in lesser exposed individuals in industrialized countries [51, 52]. During Campylobacter infection innate, cellular and humoral responses are induced but the role of these mechanisms in conferring immunity is yet unclear. Immunity appears to prevent illness but not colonization [52]. However, recent serosurveillance suggested that the majority of Campylobacter infections are asymptomatic [51]. The decreasing amount of cases due to other risk factors with age could therefore be explained with an increase in immunity. As young children are not protected, they could become ill when they are exposed to sources which harbour low Campylobacter doses, whereas elderly people only get ill when they are exposed to sources with relatively high Campylobacter doses. High prevalence in young adults could be explained by high exposure via travel and poultry pathways, where probably the highest doses of Campylobacter can be found. However, more knowledge on immunity to Campylobacter in different age groups is needed for the development of more realistic models that incorporate host susceptibility factors.

The advantage of the model presented here is the introduction of the IR per exposure, which allows combination and comparison of different dimensions of exposure (IR per portion of poultry meat, per day spent abroad, daily dog/cat contact).

As highlighted with the results of the sensitivity analysis, uncertainty of the model is mostly due to the estimation of the IR per exposure. The estimation of the IR for poultry consumption and travelling abroad were based on the results of the only one case-control study available for Switzerland with data from the year 1991 [21]. It is possible that more than 10 years later these data may have limited validity. The prevalence of Campylobacter in poultry meat in 1991 and therefore the exposure to Campylobacter through poultry consumption is unknown. Travelling behaviour, especially the choice of destinations is likely to have changed over the years which could result in a different OR for travelling abroad. However, the high OR for travelling abroad appears realistic when considering the findings of a study on the molecular 
epidemiology in Switzerland where a large proportion of human isolates did not show any similarity with isolates found in Swiss poultry, cattle or pets [25, 48], and thus could be related with infection abroad. IR for daily pet contact was based on the results of a case-control-study from Sweden, and given the cultural similarities of the two countries this approach can be justified. To obtain better estimates of the IRs, a case-control study which includes all age groups is necessary. This would also help to further elucidate the differences found in age groups and adjust the correction factors used in our model. Nevertheless, compared to data of more recent case-control studies from other countries the OR data used for our model still seem to be realistic [12, 13, 15, 18, 19, 38].

One data gap identified was in relation to underreporting of enteric diseases, and reporting behaviour in different age groups. No data were available on this for Switzerland, and we used results from studies performed abroad [8, 9, 11, 35]. Reporting behaviour needs to be better understood in order to develop accurate epidemiological assessments, not only for campylobacteriosis, but also for other diseases.

One strength of the model presented here is its flexible structure which allows the introduction of more exposure pathways when data become available. Exposure sources to consider would include ruminants and direct or indirect contact with other potential carrier animals and contaminated food as well as recreational activities, in particular if associated with surface water. There is a considerable proportion of Campylobacter cases that cannot be explained by any of the sources considered, which indicates that more research on risk factors is needed in Switzerland. Nevertheless the findings of our study can be used to inform future attempts to prevent campylobacteriosis and that risk communication strategies should account for differences in age groups.

\section{NOTE}

Supplementary material accompanies this paper on the Journal's website (http://journals.cambridge.org/ hyg).

\section{ACKNOWLEDGEMENTS}

We thank Bell AG, Zell (Dr F. Renggli and Ch. Schatzmann), Mars Schweiz AG, Switzerland (Dr J. Henner), Prodega/Growa Cash + Carry (A. Schärz), Moosseedorf and Proviande (M. Schneider),
Bern for kindly providing prevalence and consumption data, and Dr J. Danuser for his input in the early stages of the project.

\section{DECLARATION OF INTEREST}

None.

\section{REFERENCES}

1. Schmid H. Epidemiology of Campylobacter in Switzerland [in German]. Mitteilungen aus Lebensmitteluntersuchung und Hygiene 2002; 93: 24-32.

2. Wittenbrink MM. Campylobacter: prevalence and pathological significance in Humans and animals [in German]. Mitteilungen aus Lebensmitteluntersuchung und Hygiene 2002; 93: 4-8.

3. Westrell T, et al. Zoonotic infections in Europe in 2007: a summary of the EFSA-ECDC annual report. Eurosurveillance $2009 ; \mathbf{1 4}$ : pii $=19100$.

4. WHO. The increasing incidence of human campylobacteriosis. Report and Proceedings of a WHO Consultation of Experts Copenhagen, Denmark, 21-25 November 2000. Geneva, Switzerland: World Health Organization, Department of Communicable Disease Surveillance and Response; 2000.

5. FOPH (Federal Office of Public Health). Notifications of infectious diseases, week 52 [in German]. Amtliches Bulletin 2008; 1/2/3: 6-7.

6. Altekruse SF, et al. Campylobacter jejuni-an emerging foodborne pathogen. Emerging Infectious Diseases $1999 ; 5: 28-35$.

7. Scallan E, et al. Factors associated with seeking medical care and submitting a stool sample in estimating the burden of foodborne illness. Foodborne Pathogens and Disease 2006; 3: 432-438.

8. Wheeler JG, et al. Study of infectious intestinal disease in England: rates in the community, presenting to general practice, and reported to national surveillance. The Infectious Intestinal Disease Study Executive. British Medical Journal 1999; 318: 1046-1050.

9. Kuusi M, et al. Incidence of gastroenteritis in Norway - a population-based survey. Epidemiology and Infection 2003; 131 : 591-597.

10. Hall G, Raupach J, Yohannes K. An estimate of underreporting of foodborne notifiable diseases: salmonella, campylobacter and shiga toxin producing E. coli (STEC). Canberra (Australia): The Australian National University; 2006. Report No.: 0-9758409-0-8.

11. Thomas MK, et al. Estimated numbers of community cases of illness due to salmonella, campylobacter and verotoxigenic Escherichia coli: pathogen-specific community rates. Canadian Journal of Infectious Diseases \& Medical Microbiology 2006; 17: 229-234.

12. Fullerton KE, et al. Sporadic campylobacter infection in infants: a population-based surveillance case-control study. Pediatric Infectious Disease Journal 2007; 26: $19-24$. 
13. Friedman CR, et al. Risk factors for sporadic campylobacter infection in the United States: a case-control study in FoodNet sites. Clinical Infectious Diseases 2004; 38 (Suppl. 3): 285-296.

14. Neimann $\mathbf{J}$, et al. A case-control study of risk factors for sporadic campylobacter infections in Denmark. Epidemiology and Infection 2003; 130: 353-366.

15. Wingstrand A, et al. Fresh chicken as main risk factor for campylobacteriosis, Denmark. Emerging Infectious Diseases 2006; 12: 280-285.

16. Studahl A, Andersson Y. Risk factors for indigenous campylobacter infection: a Swedish case-control study. Epidemiology and Infection 2000; 125: 269-275.

17. Tenkate TD, Stafford RJ. Risk factors for campylobacter infection in infants and young children: a matched case-control study. Epidemiology and Infection 2001; 127: 399-404.

18. Unicomb LE, et al. Age-specific risk factors for sporadic campylobacter infection in regional Australia. Foodborne Pathogens and Disease 2008; 5: 79-85.

19. Danis K, et al. Risk factors for sporadic campylobacter infection: an all-Ireland case-control study. Eurosurveillance $2009 ; \mathbf{1 4}$ : pii $=19123$.

20. Rodrigues LC, et al. The study of infectious intestinal disease in England: risk factors for cases of infectious intestinal disease with Campylobacter jejuni infection. Epidemiology and Infection 2001; 127: 185-193.

21. Schorr D, et al. Risk factors for campylobacter enteritis in Switzerland. Zentralblatt für Hygiene und Umweltmedizin 1994; 196: 327-337.

22. Rosenquist H, et al. Danish strategies to control campylobacter in broilers and broiler meat: facts and effects. Epidemiology and Infection 2009; 6: 1-9.

23. Stern NJ, et al. Campylobacter spp. in Icelandic poultry operations and human disease. Epidemiology and Infection 2003; 130: 23-32.

24. Williman J, et al. Annual Report concerning foodborne disease in New Zealand 2008: Report of the Institute of Environmental Sience \& Research Limited, Christchurch, New Zealand, 2009. Client Report FW09062.

25. Pires SM, et al. Attributing the human disease burden of foodborne infections to specific sources. Foodborne Pathogens and Disease 2009; 6: 417-424.

26. Pires SM. Attributing human salmonellosis and campylobacteriosis to food, animal and environmental sources (Ph.D. thesis) Soborg, Denmark: Technical University of Denmark, Frederiksberg, Denmark: University of Copenhagen, 2009, 316 pp.

27. Samuel MC, et al. Epidemiology of sporadic campylobacter infection in the United States and declining trend in incidence, FoodNet 1996-1999. Clinical Infectious Diseases 2004; 38 (Suppl. 3): 165-174.

28. Dohoo I, Wayne M, Stryhn H. Veterinary Epidemiologic Research. AVC Inc., Charlottetown, Prince Edward Island, Canada, 2003, pp. 706.

29. Rosenquist $\mathbf{H}$, et al. Quantitative risk assessment of human campylobacteriosis associated with thermophilic campylobacter species in chickens. International Journal of Food Microbiology 2003; 83: 87-103.
30. Neff C, et al. Monitoring of zoonoses in turkey meat at retail 2005. Final report [in German]. Bern: Federal Veterinary Office, 2006, pp.17.

31. Regula G, et al. Evaluation of an antimicrobial resistance monitoring program for campylobacter in poultry by simulation. Preventive Veterinary Medicine 2005; 70: 29-43.

32. Laesser C, Bieger T. Travel Market Switzerland 2004: basic report and variables overview. St Gallen: Institute for Public Services and Tourism at the University of St Gallen, 2005, pp. 95.

33. Laesser C, Bieger, T. Travel Market Switzerland 2007: technical report and results. St Gallen: Institute for Public Services and Tourism at the University of St Gallen, 2008, pp. 49.

34. Wieland B, et al. Campylobacter spp. in dogs and cats in Switzerland: risk factor analysis and molecular characterization with AFLP. Journal of Veterinary Medicine, Series B, Infectious Diseases and Veterinary Public Health 2005; 52: 183-189.

35. Adak GK, Long SM, O'Brien SJ. Trends in indigenous foodborne disease and deaths, England and Wales: 1992 to 2000 . Gut 2002; 51: 832-841.

36. Nauta M, van der Fels-Klerx I, Havelaar A. A poultryprocessing model for quantitative microbiological risk assessment. Risk Analysis 2005; 25: 85-98.

37. Strachan NJ, et al. Attribution of Campylobacter infections in northeast Scotland to specific sources by use of multilocus sequence typing. Journal of Infectious Diseases 2009; 199: 1205-1208.

38. Stafford RJ, et al. Population-attributable risk estimates for risk factors associated with campylobacter infection, Australia. Emerging Infectious Diseases 2008; 14: 895-901.

39. Doorduyn Y, et al. Risk factors for indigenous Campylobacter jejuni and Campylobacter coli infections in The Netherlands: a case-control study. Epidemiology and Infection. Published online 12 March 2010. doi: 10.1017/S095026881000052X

40. Wilson DJ, et al. Tracing the source of campylobacteriosis. PLoS Genetics 2008; 4 e1000203.

41. Sheppard SK, et al. Campylobacter genotypes from food animals, environmental sources and clinical disease in Scotland 2005/6. International Journal of Food Microbiology 2009; 134: 96-103.

42. Mullner P, et al. Assigning the source of human campylobacteriosis in New Zealand: a comparative genetic and epidemiological approach. Infection, Genetics and Evolution 2009; 9: 1311-1319.

43. EFSA (European Food Safety Authority). Scientific Opinion on Quantification of the risk posed by broiler meat to human campylobacteriosis in the EU. EFSA Journal 2010; 8: 1-89.

44. FSA (Food Standards Agency). A critical review of interventions and strategies (both biosecurity and non-biosecurity) to reduce Campylobacter on the poultry farm. Final Report, 2008. Report No. B15025.

45. Rosenquist H, Boysen L, Bork BE. Interventions to control Campylobacter in the broiler production. 
Report of an International Expert Consultation Copenhagen. National Food Institute, Technical University of Denmark, Denmark, 2008.

46. Tam CC, et al. Chicken consumption and use of acidsuppressing medications as risk factors for Campylobacter enteritis, England. Emerging Infectious Diseases 2009; 15: 1402-1408.

47. Ekdahl K, Andersson Y. Regional risks and seasonality in travel-associated campylobacteriosis. BMC Infectious Diseases 2004; 4 : 54.

48. Wieland B, et al. Phenon cluster analysis as a method to investigate epidemiological relatedness between sources of Campylobacter jejuni. Journal of Applied Microbiology 2006; 100: 316-324.
49. EFSA (European Food Safety Authority). Opinion of the Scientific Panel on Biological Hazards on 'Campylobacter in animals and foodstuffs'. EFSA Journal 2005; 173: $1-10$.

50. Bachmann HP, Spahr U. The fate of potentially pathogenic bacteria in Swiss hard and semihard cheeses made from raw milk. Journal of Dairy Science 1995; 78: 476-483.

51. Havelaar AH, et al. Immunity to campylobacter: its role in risk assessment and epidemiology. Critical Reviews in Microbiology 2009; 35: 1-22.

52. Janssen R, et al. Host-pathogen interactions in Campylobacter infections: the host perspective. Clinical Microbiology Revues 2008; 21 : 505-518. 